
\title{
PReserch Square \\ Risk Factors Associated with Self-Removal of Shoulder Braces After Rotator Cuff Repair
}

\author{
Yoo-Sun Won \\ Chung-Ang University \\ Jae Sung Lee \\ Chung-Ang University \\ Hyoung-Seok Jung \\ Chung-Ang University \\ Ye-Hoon Jang \\ Chung-Ang University \\ Chan-Woo Jung \\ Chung-Ang University \\ Jae Yoon Kim ( $\square$ kjycje@gmail.com ) \\ Chung-Ang University
}

\section{Research Article}

Keywords: rotator cuff repair, brace, self-removal, compliance, complication

Posted Date: June 9th, 2021

DOl: https://doi.org/10.21203/rs.3.rs-586808/v1

License: (c) (1) This work is licensed under a Creative Commons Attribution 4.0 International License.

Read Full License 


\section{Abstract}

Background: Rotator cuff repair is a widely performed surgery, with the re-tear rate reportedly above $20 \%$. To protect the repair site, patients are instructed to wear braces for 4-6 weeks, but the compliance is known to be poor. This study aimed to identify the risk factors for poor compliance and to determine whether poor compliance leads to complications such as re-tears. Additionally, we planned to determine the patients' subjective factors for self-removal of the brace.

Method: This study included 101 patients who underwent rotator cuff repair between June 2019 and August 2020. The patients could select from two types of braces, namely, the sling-type and hard-type abduction brace. They were then instructed to wear the brace for 6 weeks postoperatively. During the follow-up, ultrasonography was performed to evaluate the repair site, and a questionnaire was administered to evaluate the compliance.

Result: The participants were categorized into three groups (Groups A, B, and C) according to the degree of self-removal. Compliance was found to be poor, and only 35 patients met the standard of Group A. There were no statistically significant patient factors affecting the compliance. Re-tears were noted in 4 patients. Only the surgery type (open versus arthroscopic surgery) was a statistically significant factor for re-tears. Among the subjective factors, discomfort while sleeping was the most common complaint and the most common reason for the actual removal of the brace.

Conclusion: Although the risk factors for self-removal of the braces could not be identified clearly and statistically, catastrophic re-tears requiring re-operation were observed in patients with poor compliance. For better compliance, the postoperative guidelines and education of the patients should be reviewed. Furthermore, the braces need to be modified to a more comfortable design.

\section{Introduction}

Rotator cuff repair is one of the most widely performed surgeries in the orthopedic surgery department and is associated with a good prognosis. Despite good postoperative prognosis, the re-tear rate was reportedly above 20\%. (Burkhart and Hartzler 2019; McElvany et al. 2015) Various postoperative rehabilitation protocols for wearing braces have been introduced. Abduction braces are the most widely used supports that protect the glenohumeral abduction to decrease the tension on the repaired rotator cuff. (Ghandour et al. 2019; Littlewood et al. 2019; Sgroi and Cilenti 2018; Thigpen et al. 2016; Thomson et al. 2016) However, the period for which braces should be used after surgery remains controversial among orthopedic surgeons. Some argue that early range of motion (ROM) can be helpful for the early recovery of ROM, but complications such as re-tears could occur if the time for which the brace is applied is too short. However, immobilization for an excessively long period can induce shoulder stiffness. (Denard et al. 2011; Gallagher et al. 2015; Houck et al. 2017; Parsons et al. 2010) Recent research has shown that early ROM does not affect the long-term outcome. (Gallagher et al. 2015) Therefore, recently, 
the use of braces for 4-6 weeks has been widely recommended to reduce such complications. (Burkhart and Hartzler 2019; Hsu et al. 2016)

The patient compliance with the use of braces is known to be poor. Grubhofer et al. reported that approximately $50 \%$ of the patients did not wear braces for at least $80 \%$ of the recommended time. (Grubhofer et al. 2019) Six weeks is a long time to wear braces continuously. Braces can disturb sleep, and the discomfort may increase when the brace is worn on the dominant hand. Even performing simple daily activities such as eating food can become uncomfortable and annoying. During the initial few days, patients cannot remove the braces easily owing to pain. However, 1-2 weeks after surgery, they tend to remove the braces as the pain reduces. (Grubhofer et al. 2019) Because patients usually are discharged within 1 week after surgery, more than 5 weeks of strict wearing of the brace is needed without the surveillance of the hospital. Thus, it is difficult to control the patient compliance after discharge.

Self-removal of the brace can lead to poor outcomes and complications, including re-tears.(Haering et al. 2015) Revision surgery may be needed in such cases, and if the re-tear is massive in size, arthroplasty is the only option available. Therefore, it is especially important to analyze the risk factors for poor compliance and identify ways to improve the compliance. We believe that the self-removal rate of braces could be related to factors such as age, job, type of surgery, awareness and insight of treatment, and education status. Based on these findings, we planned a study to identify the risk factors for self-removal of braces after rotator cuff repair surgery.

The purpose of this study was to analyze the risk factors for self-removal of braces, which is important for good prognosis. Furthermore, we planned to determine whether the self-removal of braces affects the re-tear rate, the patient's subjective discomfort when wearing braces, and ways to increase compliance and improve the design of the braces.

\section{li. Materials And Methods}

\section{Study design and subjects}

Participants who underwent rotator cuff repair between June 2019 and August $2020(n=101)$ at the Department of Orthopedic Surgery were enrolled. The participants had a full-thickness rotator cuff tear that was confirmed on magnetic resonance imaging, and the data were collected prospectively. At our hospital, the patients could select between two types of braces that restrict the abduction or adduction movement of the shoulder joint: sling-type brace and abduction brace. Previous studies have shown that there is no difference in the outcomes between these two braces. (Ghandour et al. 2019; Hawthorne et al. 2018; Hollman et al. 2017; Tirefort et al. 2019) Patients could select between the two braces before the surgery, and they were instructed to use the brace for 6 weeks (Figure 1).

\section{Postoperative management}


Every patient was educated on wearing the brace for 6 weeks postoperatively, before their discharge. They were instructed to wear braces at all times for 6 weeks and were allowed to remove them only when changing clothes or taking a shower. Regular follow-up was conducted at 4 weeks, 6 weeks, 3 months, and 6 months postoperatively. During the follow-up, ultrasonography was performed to evaluate the surgical site, and a questionnaire was administered at 6 weeks postoperatively to evaluate the compliance (Table 1).

\section{Assessment of data}

Questionnaires were administered to check the understanding and compliance of the patients regarding wearing the brace, and they could note down their subjective discomfort (Table 2). Questions 1, 2, 3, and 4 were designed to evaluate the patient's understanding of why they should wear braces. Questions 5, 6, 7, and 8 were designed to check the compliance. Questions 9 and 10 were short-answer questions to understand their subjective discomfort. We divided the patients into three groups (Groups A, B, and C) based on their answers to compare the compliance. Patients who visited the outpatient clinic without braces were categorized as Group C.

Additionally, patient-related factors such as sex, age, and educational status were analyzed to determine their correlation with the compliance or complications. Furthermore, the season during the day of the surgery, type of surgery, tear size, and whether surgery was performed on the dominant hand were included as probable risk factors.

The primary endpoint of this study was evaluation of the compliance and risk factors for self-removal, and the secondary endpoint was to assess whether adverse side effects such as re-tears occurred more frequently in patients with poor compliance. Finally, we aimed to understand the patients' subjective discomfort when wearing braces and find solutions for better compliance.

\section{Statistical analysis}

The Shapiro-Wilk test was used to assess the normality and confirm the distribution of the data. The difference in compliance according to the risk factor was determined using a univariate linear regression model. Descriptive statistics were used for comparisons among the groups. Independent t-tests were used for continuous variables and chi-square tests for categorical variables using Statistical Product and Service Solutions version 19.0 (IBM/SPSS; Armonk, New York); p-values $<0.05$ were considered statistically significant.

\section{lii. Results}

\section{Demographics}


In this study, 101 patients were analyzed. The sample included 55 men and 46 women, and the mean age was 64.1 years (range, 56.0-72.2 years). Among them, 66 patients underwent right shoulder surgery and 35 underwent left shoulder surgery. All 101 patients were right-hand dominant. The average body mass index was 24.6 (range, 22.0-27.2). Regarding the type of braces, 50 patients chose the sling-type brace and 51 chose the abduction brace. The distribution of the patients undergoing surgery according to the seasons was as follows: 23 in spring, 46 in summer, 6 in fall, and 26 in winter. The education status varied among the patients as follows: 31 patients had higher than college education, 30 had high-school education, and the others had lesser than middle-school education (Table 3). Except for 3 patients, all the others could accurately describe the reason for wearing the braces in the questionnaire, but there was no significant difference in the education status (Table 4).

\section{Risk factors for self-removal of the brace}

The self-removal of the brace was evaluated based on two indicators: the duration for which the brace was removed in a day and the number of times the brace was removed in a day. Patients were only allowed to remove the brace carefully when changing clothes or taking a quick shower and any reason for removal other than these were considered as self-removal. There were 20 patients who wore the brace without removal, 30 patients removed it once, 29 patients removed it 2 times, 11 patients removed it 3 times, and 11 patients removed it 4 or more times. The duration for which the brace was removed by the patient was less than 10 minutes in 56 patients, less than 30 minutes in 27 patients, less than 60 minutes in 6 patients, and over 60 minutes in 12 patients. Based on these results, the participants were categorized into three groups (Groups A, B, and C) (Table 5). Only 35 patients (35\%) met the standards of Group A (Table 6).

There were no significant differences in the self-removal time according to age, sex, body mass index, or education level. Moreover, the self-removal time did not show a significant difference according to the season in which the surgery was performed. Furthermore, differences in compliance according to the type of brace and tear size were not statistically significant.

\section{Complications}

Re-tear was confirmed on follow-up ultrasonography in 4 patients. No other complications were noted during the follow-up period. Among the 4 patients with re-tears, 1 patient belonged to Group A, 1 to Group B, and the other 2 to Group C. One patient in Group C needed reoperation, and this patient showed poor compliance. The patient stated that he did not wear the brace during most daily activities and even visited the outpatient clinic without the brace (Figure 2). However, there were no statistically significant differences in the re-tear rate among the three groups (Table 7). The tear size was not a significant factor for the occurrence of re-tears (Table 8). The only statistically significant factor for re-tears was the surgery type. Patients who underwent open surgery showed a significantly higher risk of re-tears than those who underwent arthroscopic surgery (Table 9). 


\section{Subjective factors of self-removal of the brace}

The last two questions in the questionnaire were about the patient's subjective discomfort when wearing the brace. The subjective discomfort was similar between the two brace types. More than half of the patients answered that they felt most uncomfortable while sleeping. Irritation in the arm and neck was the second most common cause of discomfort, followed by heat sensation and improper size of the brace (Table 10). The actual circumstances in which the patients removed the brace were similar to these discomfort causes reported by the patients. Twenty-seven patients removed the brace when sleeping, eleven removed it because of irritation to the arms and neck, and nine removed it for pain relief (Table 11).

\section{Iv. Discussion}

There have been several discussions regarding the importance of protecting the surgical site by wearing a brace after rotator cuff repair, and a lot of research has been conducted on how long the patients should wear the brace. (Denard et al. 2011; Gallagher et al. 2015; Houck et al. 2017; Parsons et al. 2010) Furthermore, significant research has been conducted on the rehabilitation plans after surgery. (Denard et al. 2011; Gallagher et al. 2015; Thigpen et al. 2016) However, there is limited discussion about the postoperative management in terms of patient compliance, and whether patients wear the brace as trained at the hospitals. This means that the true effect of braces is hypothetical. In one study, a heat sensor was installed on the brace to analyze the patient's actual compliance, and it was confirmed that the compliance was much lower than the self-reported time. (Grubhofer et al. 2019) In our research, only $35 \%$ of the patients met the standard of Group A, indicating that the general compliance of wearing braces is much lower than expected. Therefore, when assessing guidelines for postoperative management, it should be considered that the actual compliance would be much lower than expected.

Based on the abovementioned observation, the guidelines for braces need to be reconsidered. At our hospital, we instruct the patients to wear the brace all the time for 6 weeks, except when they change clothes or take a shower. Despite poor compliance, re-tears were reported only in 4 patients. This result implies that mitigating the guidelines can be possible, such as allowing patients to remove the brace when they can control and limit shoulder movement carefully during simple daily activities.

This study did not reveal any statistically significant risk factors for re-tears, except for the type of surgery. Open surgery was the only statistically significant risk factor for re-tears. However, this does not directly imply that the open surgical technique itself is a risk factor. Open surgery was indicated in patients with rotator cuff tears, which seemed difficult to repair with the arthroscopic technique. Thus, the characteristics of the tear itself could affect the results. However, the tear size did not show a significant relationship with the occurrence of re-tears in this study. Additionally, self-removal time was not a statistically significant risk factor for re-tears, and there was no significant difference in self-removal time among the three groups. However, there was one patient with severe re-tears requiring reoperation in Group C, and he almost never wore the brace after discharge. Moreover, he did not bring the brace when 
visiting the outpatient clinic for follow-up. This indicates that poor compliance can cause catastrophic results in patients; hence, wearing the brace to protect the surgical site is important.

Compliance with wearing the brace was not related to the patient's educational status or sex. Even though the patients were well aware of the importance and reasons for wearing the brace, only $35 \%$ of them wore it as instructed. This shows that awareness and education status might not be the most important factors for compliance. Therefore, other factors that cause patients to remove the brace, regardless of their awareness, should be noted, such as the patient's subjective discomfort.

This study focused on the patients' subjective discomfort. Initially, abduction braces, which are made of hard materials, were thought to be more uncomfortable than the sling-type brace. However, there was no difference in the compliance between the two braces. Furthermore, the discomfort reported by the patients was similar between the two braces. The most common discomfort reported was during sleeping, and the actual removal of the brace was done mostly when sleeping. Patients complained that the brace itself was too bulky and difficult to position while sleeping, and it was the most common complaint with both the brace types. New and modified types of braces could be the solution to this problem, but it is difficult to design comfortable and efficient new braces immediately. Thus, simple nighttime braces could be the solution. In the knee department of our hospital, patients who undergo anterior cruciate ligament reconstruction additionally use a detachable lightweight splint when sleeping, because the cruciate ligament braces are bulky and difficult to wear. In patients undergoing rotator cuff surgery, it may be helpful to develop an additional lightweight brace for nighttime, such as a simple sling brace without an abduction pillow.

Other discomfort reported by the patients included irritation in the neck or armpits, heat sensation when wearing the brace, and improper size of the brace, which caused the patients to remove the brace. Additionally, there was an interesting point regarding the reasons for self-removal. Nine patients answered that they took off the brace because their pain had relieved. This shows that not only pain and discomfort, but also pain relief can cause patients to be less alert and remove the brace. This part should be emphasized on when educating the patients on the importance of wearing the brace. It should be explained to the patients that pain relief does not mean that the repaired cuff has healed.

This study has some limitations. First, the patient compliance was analyzed based on a questionnaire that was filled out subjectively by the patient. It was impossible to determine the compliance objectively and accurately after the patient was discharged from the hospital. Therefore, the questionnaire evaluated the compliance based on the patients' answers. To overcome the limitations of subjective data, we attempted to design the questionnaire in the most efficient form. We used simple and easy terms in the questionnaire for the elderly patients because too many requirements and complexities in the questionnaire could have distorted the results.

Second, although the questionnaire evaluated for the time and frequency of the brace removal, it was difficult to assess how the patients behaved after removing the brace. For example, some patients may have behaved carefully after the brace removal, while others may have used their arms roughly. 
Therefore, the same amount of removal time in patients does not indicate the same degree of compliance.

Third, only one size of each brace was available for the patients. Even though braces can be adjusted to some extent, they may not fit all patients appropriately. Therefore, the same brace may fit differently in different patients, and this could be one of the factors causing discomfort to the patient. Availability of the brace in various sizes could help in reducing the discomfort in patients having trouble with ill-fitting braces.

Fourth, ultrasonography was used to evaluate the rotator cuff only postoperatively. Although current research has shown that ultrasonographic examination of the rotator cuff is comparable to magnetic resonance imaging, it remains subjective to the examiners. (Lee et al. 2018) In our study, we tried to increase the objectivity by saving the images in our database to be re-checked if needed.

\section{Conclusion}

This study was conducted at our institute to investigate the patient compliance in wearing the brace and to identify the risk factors for poor compliance. Furthermore, we attempted to determine the subjective factors affecting poor compliance. We found that the compliance was much lower than expected. During the 6 weeks of strict brace usage, the patients complained about sleeping difficulty, irritation around the armpit or neck, heat sensation, and improper size of the brace.

Although the risk factors for self-removal of the braces were not identified clearly and statistically, catastrophic re-tears requiring re-operation were noted in patients with poor compliance. For better compliance, the postoperative guidelines and education of patients should be reviewed. Furthermore, modifying the braces to a more comfortable design is needed.

\section{Declarations}

\section{Ethics approval and consent to participate}

The study protocol was approved by Institutional Review Board of Chung-Ang University Hospital (IRB No. 1982-003-384). Every informed consent was obtained from patients and no subjects were under 18. All methods were carried out in accordance with relevant guidelines and regulations.

\section{Consent for publication}

Consent for publication is given by all authors.

\section{Availability of data and material}

Details of the data are provided in the additional files section and can be obtained by the corresponding author via personal communication. 


\section{Competing interests}

The author(s) declared no potential conflicts of interest with respect to the research, authorship, and/or publication of this article.

\section{Funding}

The author(s) received no financial support for the research, authorship, and/or publication of this article.

\section{Authors' contributions}

Yoo-Sun Won and Jae-Yoon Kim wrote the main manuscript text.

Jae-Sung Lee and Hyoung-Seok Jung conceptualized main ideas and analyzed datas and Ye-Hoon Jang and Chan-Woo Jung prepared figures.

All authors reviewed the manuscript.

\section{Acknowledgements}

The authors in this study express our sincere appreciation for all the patients to join this study.

\section{References}

1. Burkhart SS, Hartzler RU. 2019. Arthroscopic rotator cuff repair: How to avoid retear. Arthroscopy. 35(1):12-13.

2. Denard PJ, Ladermann A, Burkhart SS. 2011. Prevention and management of stiffness after arthroscopic rotator cuff repair: Systematic review and implications for rotator cuff healing. Arthroscopy. 27(6):842-848.

3. Gallagher BP, Bishop ME, Tjoumakaris FP, Freedman KB. 2015. Early versus delayed rehabilitation following arthroscopic rotator cuff repair: A systematic review. Phys Sportsmed. 43(2):178-187.

4. Ghandour TM, Ibrahim A, Abdelrahman AA, Elgammal A, Hammad MH. 2019. Does the type of shoulder brace affect postoperative pain and clinical outcome after arthroscopic rotator cuff repair? Arthroscopy. 35(4):1016-1023.

5. Grubhofer F, Gerber C, Meyer DC, Wieser K, Ernstbrunner L, Catanzaro S, Bouaicha S. 2019. Compliance with wearing an abduction brace after arthroscopic rotator cuff repair: A prospective, sensor-controlled study. Prosthet Orthot Int. 43(4):440-446.

6. Haering D, Blache Y, Raison M, Begon M. 2015. Mechanical risk of rotator cuff repair failure during passive movements: A simulation-based study. Clin Biomech (Bristol, Avon). 30(10):1181-1188.

7. Hawthorne JR, Carpenter EM, Lam PH, Murrell GAC. 2018. Effects of abduction pillows on rotator cuff repair: A biomechanical analysis. HSS J. 14(2):114-122. 
8. Hollman F, Wolterbeek N, Zijl JAC, van Egeraat SPM, Wessel RN. 2017. Abduction brace versus antirotation sling after arthroscopic cuff repair: The effects on pain and function. Arthroscopy. 33(9):1618-1626.

9. Houck DA, Kraeutler MJ, Schuette HB, McCarty EC, Bravman JT. 2017. Early versus delayed motion after rotator cuff repair: A systematic review of overlapping meta-analyses. Am J Sports Med. 45(12):2911-2915.

10. Hsu JE, Horneff JG, Gee AO. 2016. Immobilization after rotator cuff repair: What evidence do we have now? Orthop Clin North Am. 47(1):169-177.

11. Lee SC, Williams D, Endo Y. 2018. The repaired rotator cuff: Mri and ultrasound evaluation. Curr Rev Musculoskelet Med. 11(1):92-101.

12. Littlewood C, Bateman M, Cooke K, Hennnings S, Cookson T, Bromley K, Lewis M, Funk L, Denton J, Moffatt $\mathrm{M}$ et al. 2019. Protocol for a multi-centre pilot and feasibility randomised controlled trial with a nested qualitative study: Rehabilitation following rotator cuff repair (the racer study). Trials. 20(1):328.

13. McElvany MD, McGoldrick E, Gee AO, Neradilek MB, Matsen FA, 3rd. 2015. Rotator cuff repair: Published evidence on factors associated with repair integrity and clinical outcome. Am J Sports Med. 43(2):491-500.

14. Parsons BO, Gruson KI, Chen DD, Harrison AK, Gladstone J, Flatow EL. 2010. Does slower rehabilitation after arthroscopic rotator cuff repair lead to long-term stiffness? J Shoulder Elbow Surg. 19(7):1034-1039.

15. Sgroi TA, Cilenti M. 2018. Rotator cuff repair: Post-operative rehabilitation concepts. Curr Rev Musculoskelet Med. 11(1):86-91.

16. Thigpen CA, Shaffer MA, Gaunt BW, Leggin BG, Williams GR, Wilcox RB, 3rd. 2016. The american society of shoulder and elbow therapists' consensus statement on rehabilitation following arthroscopic rotator cuff repair. J Shoulder Elbow Surg. 25(4):521-535.

17. Thomson S, Jukes C, Lewis J. 2016. Rehabilitation following surgical repair of the rotator cuff: A systematic review. Physiotherapy. 102(1):20-28.

18. Tirefort J, Schwitzguebel AJ, Collin P, Nowak A, Plomb-Holmes C, Ladermann A. 2019. Postoperative mobilization after superior rotator cuff repair: Sling versus no sling: A randomized prospective study. J Bone Joint Surg Am. 101(6):494-503.

\section{Tables}

Table 1. Postoperative follow-up schedule 


\begin{tabular}{|lllll|}
\hline Rehabilitation & $\begin{array}{l}\text { POD \#4 } \\
\text { weeks }\end{array}$ & $\begin{array}{l}\text { POD \#6 } \\
\text { weeks }\end{array}$ & POD \#3 months & POD \#6 months \\
& $\begin{array}{l}\text { Start passive } \\
\text { ROM }\end{array}$ & $\begin{array}{l}\text { Brace off } \\
\text { Start active } \\
\text { ROM }\end{array}$ & $\begin{array}{l}\text { Start muscle strengthening } \\
\text { exercises }\end{array}$ & $\begin{array}{l}\text { Start all sports } \\
\text { activities }\end{array}$ \\
Ultrasonography & $\square$ & $\square$ & $\square$ \\
Questionnaire & $\square$ & & \\
\hline
\end{tabular}

(POD, postoperative day; ROM, range of motion)

\section{Table 2. Questionnaire}


1. Why did you undergo surgery? (Short-answer question)

2. What type of surgery did you undergo?

1) Rotator cuff repair

2) Shoulder arthroplasty

3) Others (

3. Why should you wear a brace? (Short-answer question)

4. How long should you wear the brace?

1) 2 weeks

2) 4 weeks

3) 6 weeks

4) 8 weeks

5. When did you stop wearing the brace?

1) 2 weeks after surgery

2) 4 weeks after surgery

3) 6 weeks after surgery

4) 8 weeks after surgery

6. How many times did you remove the brace in a day?

1) 1 time or less

2) 2 times

3) 3 times

4) 4 times or more

7. For how long did you remove the brace in a day?

1) Less than 10 minutes

2) Less than 30 minutes

3) Less than 60 minutes

4) More than 60 minutes

8. How did you wear the brace? (Short-answer question)

9. What was the most uncomfortable part of wearing the brace? (Short-answer question)

10. Why did you remove your brace before 6 weeks? (Short-answer question) 
Table 3. Demographic characteristics of the participants $(n=101)$

\begin{tabular}{|ll|}
\hline Sex (female/male) & $46 / 55$ \\
\hline Age (years) & $64.1(56.0-72.2)^{*}$ \\
\hline Right/Left shoulder & $66 / 35$ \\
\hline Dominant/non-dominant shoulder & $66 / 35$ \\
\hline Body mass index & $24.6(22.0-27.2)^{*}$ \\
\hline Season during the day of the surgery & \\
\hline \multicolumn{1}{|c|}{ Spring } & 23 \\
\hline \multicolumn{1}{|c|}{ Summer } & 46 \\
\hline \multicolumn{1}{|c|}{ Fall } & 6 \\
\hline \multicolumn{1}{|c|}{ Winter } & 26 \\
\hline Surgery type & \\
\hline Arthroscopic surgery & 91 \\
\hline Open surgery & 10 \\
\hline Type of brace & 30 \\
\hline Ultra-Sling (sling type) & 50 \\
\hline Shoulder abduction brace & 51 \\
\hline Educational status (college degree/no college degree) \\
\hline College graduate or higher & 31 \\
\hline High school graduate & 30 \\
\hline Middle school graduate or lesser & \\
\hline Complications (Re-tears) & \\
\hline Re-operation & \\
\hline
\end{tabular}

$\star$, mean (range: \pm SD)

Table 4. Patients' answers for the reason for wearing braces 


\begin{tabular}{|ll|}
\hline Reason & $\mathrm{N}$ \\
\hline To protect the surgical site & 98 \\
\hline No answer & 3 \\
\hline
\end{tabular}

Table 5. Classification of the participants

\begin{tabular}{|lllll|}
\hline Number of times & 1 time or less & 2 times & 3 times & 4 times or more \\
\hline Time & & & & \\
\hline$<10$ min & Group A & Group A & Group B & Group C \\
\hline$<30$ min & Group A & Group B & Group B & Group C \\
\hline$<60$ min & Group B & Group B & Group B & Group C \\
\hline 60 min & Group C & Group C & Group C & Group C \\
\hline
\end{tabular}

(Time : Time for which the brace was removed in a day, Number of times: Number of times the brace was removed in a day)

Table 6. Patient distribution among the groups

\begin{tabular}{|ll|}
\hline & N \\
\hline Group A & 35 \\
\hline Group B & 35 \\
\hline Group C & 31 \\
\hline
\end{tabular}

Table 7. Re-tear rates of the groups

\begin{tabular}{|llll|}
\hline & No re-tear & Re-tear & p-value \\
\hline Group A & 34 & 1 & 0.694 \\
\hline Group B & 34 & 1 & \\
\hline Group C & 29 & 2 & \\
\hline Total & 97 & 4 & \\
\hline
\end{tabular}

$\star *$, Statistically significant $(p<0.05)$

Table 8. Tear size and re-tear rate 


\begin{tabular}{|llll|}
\hline & No re-tear & Re-tear & p-value \\
\hline Small & 27 & 1 & 0.345 \\
\hline Medium & 35 & 0 & \\
\hline Large & 27 & 2 & \\
Massive & 8 & 1 & \\
Total & 97 & 4 & \\
\hline
\end{tabular}

**, Statistically significant $(p<0.05)$

Table 9. Surgery type and re-tear rate

\begin{tabular}{|llll|}
\hline & No re-tear & Re-tear & p-value \\
\hline Open surgery & 8 & 2 & $0.006^{\star \star}$ \\
\hline Arthroscopic surgery & 89 & 2 & \\
\hline Total & 97 & 4 & \\
\hline
\end{tabular}

**, Statistically significant $(p<0.05)$

Table 10. Discomfort reported by the patients when wearing the brace

\begin{tabular}{|ll|}
\hline Factors & N \\
\hline When sleeping & 62 \\
\hline Irritation in the armpits or neck & 14 \\
\hline Heat sensation & 11 \\
\hline Improper size & 4 \\
\hline
\end{tabular}

Table 11. Reasons for self-removal of the brace

\begin{tabular}{|ll|}
\hline Reasons for removal & N \\
\hline Discomfort when sleeping & 27 \\
\hline Painful to wear the brace & 16 \\
\hline Took it off as pain had relieved & 9 \\
\hline Too hot to wear the brace & 4 \\
\hline
\end{tabular}


Figures

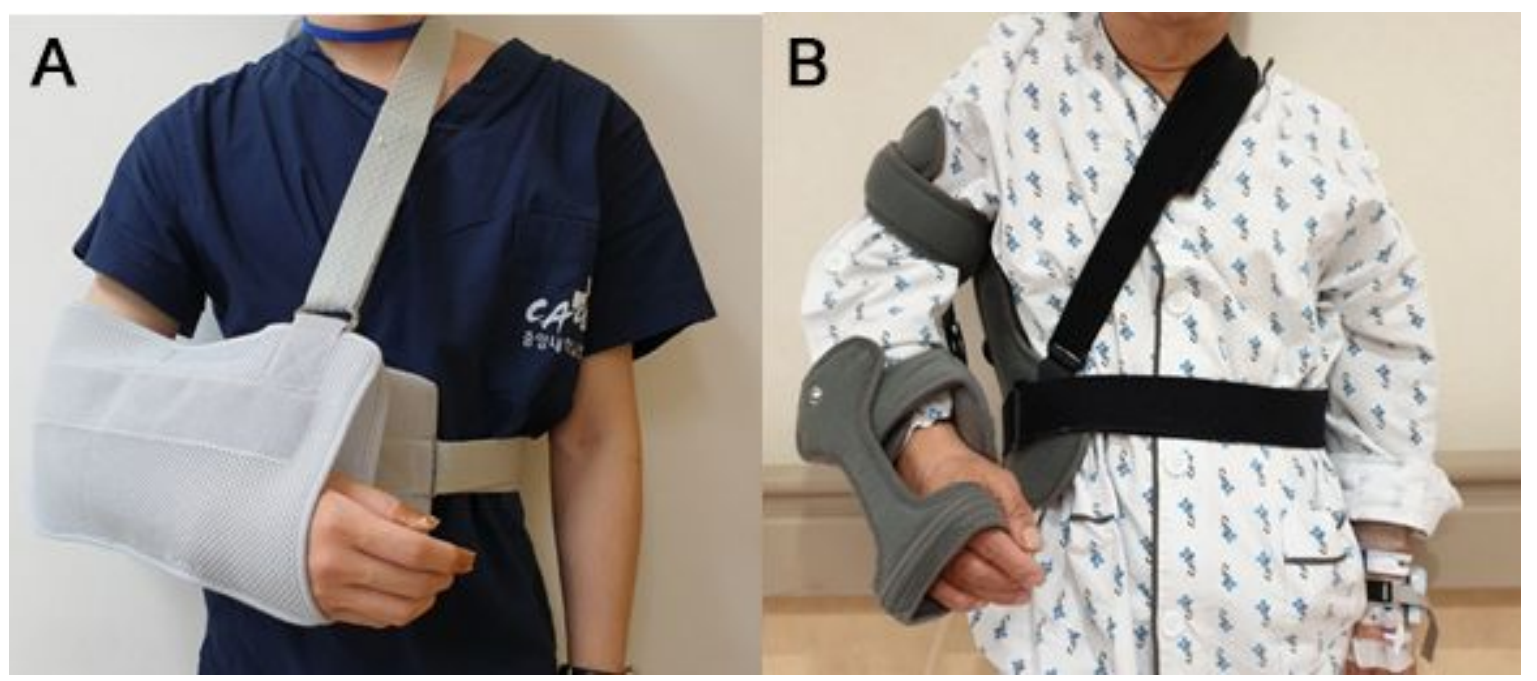

Figure 1

Type of braces (A: Sling-type brace; B: Abduction brace)
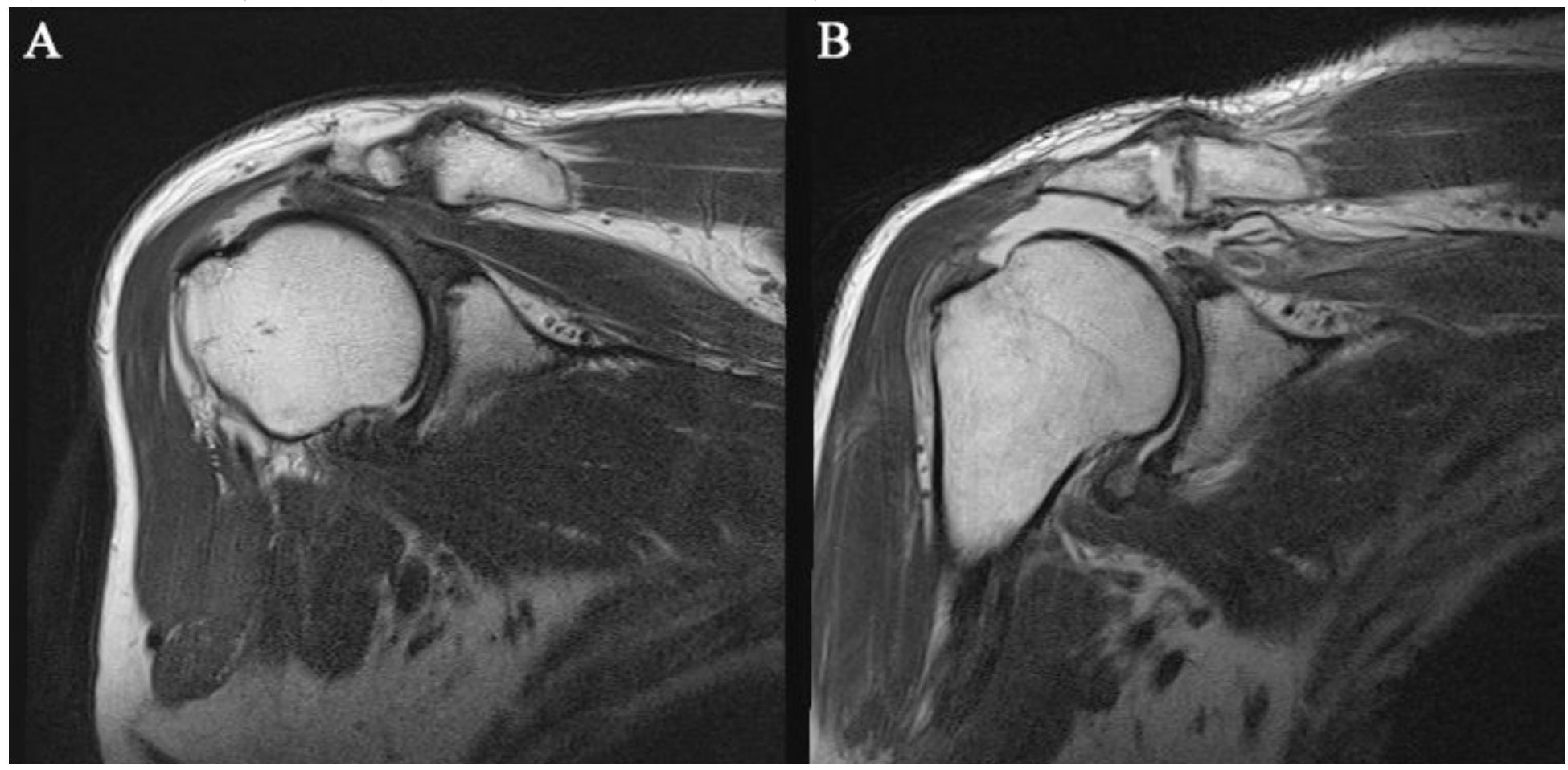

Figure 2

Preoperative and postoperative magnetic resonance imaging of patient with re-tears A: Preoperative MRI image; B: Postoperative MRI image (MRI was performed for re-operation in this patient) MRI, magnetic resonance imaging 